GENERAL DYNAMICS IN OVERLAPPING

\title{
GENERATIONS MODELS*
}

Carmen Carrera

University of British Columbia and

Universidad Complutense de Madrid

Manuel Morán

Instituto Complutense de Análisis Económico

Universidad Complutense

Campus de Somosaguas

28223 Madrid

ABSTRACT

In this article we analyse the dynamics generated by the equilibrium solution in an overlapping generations model with production.we adopt an inverse approach to characterise and construct the class of economies that generates any twice continuously differentiable dynamics. To do this we introduce a any twice continuously differentiable dynamics. To do this we
technique based on the theory of partial differential equations.

\section{RESUMEN}

Se analiza en este artículo las dinámicas generadas por las soluciones de equilibrio en un modelo de generaciones sucesivas con producción. El punto de vista adoptado es el inverso. Es decir, se parte de una dinámica dos veces diferenciable cualquiera, y se caracterizan y se construyen las clases de economías que generan esta dinámica. Se prueba que dinámicas arbitrariamente caóticas pueden ser generadas por modelos convencionales. Para conseguir estos resultados, se introduce una técnica basada en las ecuaciones diferenciales en derivadas parciales. 


\section{INTRODUCTION}

In this article we solve the inverse problem for the overlapping generations $(O G)$ model. We take as given any $\mathrm{C}^{2}$ dymamics and a production function and search for the utility functions compatible with those parameter functions.

We give necessary and sufficient conditions characterising a utility function capable of generating the given dynamics, thus solving in a complete manner the 1nverse problem. The closest precedent of our work is that of Boldrin and Montrucchio (1986) who gave sufficient conditions for a standard growth model to generate a given $\mathrm{C}^{2}$ dynamics.

The OG model provides a general scheme which has been widely used to analyse the consequences of economic policies in a dynamic setting. The first natural question to settle is that of determining the conditions of existence and properties of an equilibrium. This was thought by early researchers to be preeminently a stationary solution of the model in the ine that was standard in static models. The growing understanding of non linear dynamics over the last decade led economists to enquire about the possibility that deterministic economic systems may generate complex dynamics, thus providing an endogenous explanation of cycles or chaos. The theorem by Li and Yorke (1975) proving that the existence of a cycle of period three implies chaos was used by Benhablb and Day (1982), Grandmont (1985) and other authors to show that an entirely conventional OG model may undergo endogenous fluctuations of any periodicity, a property usually thought to be linked to chaotic behavior. This leads to what ought to be a natural goal for dynamic analysis: to find the relationship between classes of economies and classes of dynamics without ruling out a priori any form of behavior which can be derlved from what are considered conventional 
assumptions on the utility and production functions. A major step in this direction is the solution of the inverse problem. By this we mean a setting up of the problem which starts with any specific dynamics as a datum and constructs the economies capable of generating it, thus opening the way to a complete analysis of the structural relationships among the economic categorles of the model. This is a more comprehensive analysis than a simple proof that a particular kind of behavior, for instance chaotic behavior, can be standard for certain models. But even. from the point of view of the legitimacy of chaotic behavior, the solution of the inverse probletn proves to be a truly satisfactory positive answer. In fact, to prove the existence of chaos in the sense of $\mathrm{LI}$ and Yorke for a dynamics $\mathrm{h}$ requires the proof that $h^{3}(x) \leq x<h(x)<h^{2}(x)$ holds for some $x$. This condition is quite simple and allows the characterization of fairly neat sufficient conditions for 1t. 'In turn 1t only guarantees chaos in a weak sense, namely the existence of periods of all orders. However it is well known that all these cycles can be repelling, and for this reason unobservable, with the exception of a unique attracting cycle of period three, in such a way that a path randomly chosen (for example with a uniform probability distribution for its initial condition) will converge to the stable period three cycle with probability one. Moreover, this behavior may well be structurally stable. However the solution of the inverse problem shows the possibility of dynamics with chaotic paths of positive probability. Furthermore such dynamics can be proved to have positive probability in a suitable parameter space - see Falconer (1990).

Inverse problems are not new in economics. Debreu-(1974) and Mantel (1974) found classes of preferences compatible with excess demand functions exhibiting given properties. In the field of growth theory the article by Boldrin and Montrucchio showed that any dynamics is compatible with a conventional capital theory model. Although Sorger (1992) found a necessary condition showing that the result of Boldrin and Montrucchio only stands for high rates of discount, thelr article contributed significantly to emphasize the 1nterest of inverse problems.

Our approach to the inverse problem for OG permits, for the first time in the 1iterature, the obtention of necessary and sufficlent conditions characterising the economies generating a particular dynamics. It turns out that such conditions are fulfilled by a wide family of conventional economies that can be parameterised by the set of all non negative $C^{2}$ functions defined in a certain domain of the plane. These results show that the information about individual preferences that can be extracted from an observed dynamics is essentially local. Behind this fact there is a topological structural reason which is common to all OG models. A dynamics imposes conditions over a manifold of smaller dimension than that of the domain of the utility function. So the dymamics gives information only about the preferences in a set of points whose complement is open and dense in the domain of the utility function. However we obtain a natural criterion to classify consumer preferences related to the elasticity of substitution which, for a given production function, allows us to establish a bijective correspondence between economies and dynamics.

In the model we use here there is a striking contrast between the flexibillty to generate any given dynamics from wide families of different utility functions and the result obtained using the direct approach where a unique dynamics is completely determined by a given economy. Since this is a 
direct consequence of the structural character of $O G$ models, we may expect it to be a fairly general property of such models.

When we think of the inverse approach to a differentiable optimisation problem, that is when attempting to characterise the objective function generating given solutions, the first and second order conditions are restrictions on the partial derivatives of the unknown function. This is also the case when imposing the usual convexity assumptions on consumer preferences. Thus the natural tool to solve this type of problen is the theory of partial differential equations. Thls has been our basic technique in this article. We believe this theory to be very powerful in dealing adequately with inverse dynamic problems.

The article is organized as follows. In the first section we present the model and solve the direct problem. In the second section we solve the inverse problem. Lastly we present some conclusions and the proofs of the theorems.

2. DECISION MODEA.

We study the dynamic behavior over time of an overlapping generations economy composed of identical indfviduals and firms. There is no uncertainty in the model. Production is carried out by identical firms using a constant returns to scale technology represented by a homogeneous function $F(L(t), x(t))$ of degree one, where $L(t)$ and $x(t)$ are the levels of labor and capital used in period $t$. Without any loss of generality we treat all firms as one. We assume no depreciation. The level of labor used each period is normalized to $L(t)=1$ for every $t$. We define the production function $f(x(t))$ as $f(x(t))=F(1, x(t))$. He assume that each firm takes the prices of output $p(t)$, labor $w(t)$ and cap1tal $r(t)$ as $g$ iven in each perlod. We normalize the price of output $p(t)=1$ for every perlod. The firm uses each input in such a way that the value of 1 ts marginal product equals the price of the input in that perlod. Total output is distributed to the providers of labor. and capltal in proportion to their marginal productivities. That is, using Euler's Theorem, we can write

$$
f(x(t))=x(t) f^{\prime}(x(t))+w(t)=x(t) r(t)+w(t) .
$$

We assume each individual lives for two consecutive periods. The number of Individuals is the same in every period. For simplicity we treat then all as one. He is endowed with a unit of labor in the first period of his life, which he supplies inelastically in production, having no regard for leisure. Production in the first period of life of an individual born in $t$ is accomplished using his labor together with a capital, provided by his parent, which equals the value $x(t)$ of output saved in the previous perlod. The salary given in (1) must provide for the individual consumption and saving when young while the gross return $x(t+1)(1+r(t+1))$ on his savings will cover the value of his consumption when old. For ease of notation we will from now onwards denote by $x$ the optimal choice $x(t+1)$ of the individual savings when young and we will call $z$ the savings choice made by his parent. Since the value of $z$ is taken as given by the individual we characterise his choice of $\mathrm{x}$ by the solution of the following constralned optimisation problem which we denote by $\mathrm{P}_{z}$. 


$$
\left\{\begin{array}{l}
P_{z} \equiv \operatorname{Max}_{x} U\left(c_{1}(t), c_{2}(t)\right) \\
\text { subject to } \\
c_{1}(t)=f(z)-z f^{\prime}(z)-x \\
c_{2}(t)=x\left(1+f^{\prime}(x)\right) \\
q_{1}(z)<x \leq \sigma_{2}(z)
\end{array}\right.
$$

Where $U\left(c_{1},(t), c_{2}(t)\right)$ is a Utillty function representing the preferences of the consumer born in $t$ about first and second pertod consumption. The bounds $\sigma_{1}(z)$ and $\sigma_{2}(z) 11 \mathrm{~m} ! \mathrm{t}$ the feasible values of saving to ensure, for instance, that consumption in both periods remalns positive. since the optimal value of $x$ is always chosen glven $z$ and $1 \mathrm{n}$ every period an individual is born, and solves this type of problen, we have a family of one variable optimisation problems parameterised by every possible value of $z$. We represent in figure 1,2 typlcal sequence of budget constraints and its associated optimal consumption points belonging to a curve $\Psi(\Gamma)$ representing optimal consumptions (see Lemma below).

$$
\text { Figure } 1 .
$$

We now formulate several assumptions to ensure that, for each possible value of $z, P_{z}$ has a well defined unique solution.

We consider $\mathrm{z}$ taking values over an interval $\mathrm{I} S \mathbb{R}^{+}$, Let

$$
\Lambda_{z}=\left\{\left(c_{1}(z, x), c_{2}(x)\right): x \in I_{z}\right\},
$$

where $I_{z}=\left\{x: \sigma_{1}(z) \leq x \leq \sigma_{2}(z)\right\}$, be the feasible set for the problem $P_{x}$. The character of the production function ensures, given (2) y (3), a specific shape for the budget constraint. We make the following assumptions on $f$

\section{ASSLMPTIONS ON $f$}

The production function $f: I \longrightarrow \mathbb{R}$ fulfills:

1) $f>0, f^{\prime}>0, f^{\prime \prime}<0$.

ii) $A(x)=1+f^{\prime}+x f^{\prime \prime}>0$.

(iii) $f^{\prime \prime \prime} \leq 0$.

We prove in Theorem 2 below that there exists a wide famlly of production functions verifying requirements i) to 1ii) together with another condition we refer to at the beginning of section three. Assumptions 1), 1i), and 1ii) guarantee that the curve $\Lambda_{z}$, representing the feasible set for the problem $P_{z}$, is the graph of a decreasing, strictly concave function $c_{2}=$ $R\left(c_{1}\right)$ in the consumption space. Observe that equation (2) shows that the correspondence between $c_{1}$ and $x$ is bijective. Therefore a given value of $c_{1}$ determines a unique value of $x$ and, given (3), a unique value of $c_{2}$. Also $R^{\prime}\left(c_{1}\right)=-1-f^{\prime}(x)-x f^{\prime \prime}(x)<0$ and $R^{\prime \prime}\left(c_{1}\right)=2 f^{\prime \prime}(x)+x f^{\prime \prime}(x)<0$, where $x=f(z)-z f^{\prime}(z)-c_{1}$

We now make the following assumptions on the utility function ASSUMPTIONS ON U.

a) $U$ is defined on $D=U_{z \in I} D_{z}$, where each $D_{z}$ is an open convex set such that $\Lambda_{\mathbf{z}} \subseteq D_{\mathbf{z}}$.

b) $u=c^{2}(n)$

c) $U_{1}>0, i=1,2$, where $U_{1}$ denotes the first order derivatives of $U$.

d) The restriction of $U$ to each $D_{2}$ is a quasi concave function.

e) For every $z \in I$ there exists a point $x$ verifying the first order condition (6) below, with $\sigma_{1}(z)<x<\sigma_{2}(z)$.

Notice that in order to apply the usual convex programming techniques for each $P_{z}$ we do not need $D$ to be a convex set. Furthermore when we deal 
with the inverse problem we will not be able to assume that $D$ is convex. Thus we make no assumptions on the convexity of $\mathrm{D}$. Assumption e) ensures the existence of an interlor solution for each $P_{z}$. A natural sufficient condition for the existence of a point fulfilling e) would be $-U_{1}+$ $U_{2} A\left(\sigma_{1}(z)\right)<0$ and $-U_{1}+U_{2} A\left(\sigma_{2}(z)\right)>0$, since by continuity, for some $x \in I_{z}$ (6) would hold. In section three, theorem 4, we prove the existence of wide familles of functions $U$ fulfilling a) to e).

The first order condition for the problem $P_{z}$ is

$$
-\mathrm{U}_{1}+\mathrm{U}_{2} \mathrm{~A}(\mathrm{x})=0
$$

Under these conditions we can use standard elementary techniques in convex programing to prove the following theorem.

THEOREM 1. Under assumptions i) to iii) on $f$ and a) to d) on $U$ above the necessary first order condition for the problem $P_{z}$ is also a sufficient condition for a global and unique maximsam. Moreover if assumption e) on $U$ holds such maximum always exists.

Hence, 8 iven assumptions a) to d) on $U$, every point $x$ satisfying (6) can be written as

$$
x=h(z)
$$

The dynamic nature of the problem 1 s expressed by the fact that the opt1mal value of savings in period $t, x=h(z)$, is the value of capital for the foll lowing generation, that 15 , the parameter defining the problem $P_{h(z)}$. Hence if for every 2 there 15 a value of $x$ satisfying the first order condition, $\mathrm{h}$ expresses the optimal recurrence for the model. As an 11lustration, figure 2 below shows an example of a possible dynamics for (7) together with its translation into consumption values.
Assume now that assumption e) holds. Given theorem 1 it will also satisfy the second order condition for $\mathrm{P}_{z}$, namely

$$
U_{11}-2 U_{12} A(x)+A(x)^{2} U_{22}+U_{2} A^{\prime}(x)<0
$$

Therefore equation (6) above defines 1raplicitly the function $x=h(z)$. In order to obtain information about $h(z)$ we differentiate the first order conditions. We then get

$$
\frac{d x}{d z}=\frac{-z f^{\prime \prime}(z)\left(U_{11}-A(x) U_{21}\right)}{U_{11}-2 U_{12} A(x)+A(x)^{2} U_{22}+U_{2} A^{\prime}(x)} .
$$

Given (8) the sign of this derivative depends on the sign of the numerator. Our assumptions on $U$ and $f$ allow any sign for it. In particular if it were to be the case that $U_{12}=0$ we would have simple dynamics, while if $U_{12}<0$ we would be allowing for the possibility that $d x / d z$ might change sign continuously and, in consequence, the dynamics described by (7) might be complex. We point out that Benhabib and Nishimura (1985) show that, for a model with a representative agent living an infinite number of periods, $v_{12}$ $>0$. Implies that the optimal path is monotonic while $U_{12}<0$ allows oscillatory dynamics.

This suggests the possibility of analysing the inverse problem. What

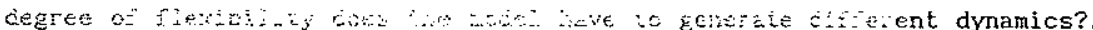
Can we fix an arbitrary dynamics for the model which is compatible with conventional utility and production functions?. We do this in the next Section. 
We consider a mapping $h: I \rightarrow$ I from the domain of $f, I=\left(x_{0}, x_{1}\right) \subseteq R^{*}$, onto itself. This mapping gives us the dynamics which we want to impose on the optimal path in this model. The only assumption that we make on $h$ is

ASSUMPTION 1): $h \in C^{2}(I)$.

Notice that this assumption allows an extremely wide range of simple or complex dynamics, from monotone to multimodal functions.

We now make the extra assumption on $f$ we referred to in section two when we listed the requirements on the production function we needed there.

ASSUMPTION IV) ON $\mathrm{f}:-z f^{\prime \prime}-h^{\prime}>0$.

We now p1ck a production function fulfilling assumptions i) to Iv) on $f$. Notice that since

$$
\left.\frac{d c}{d z}\right|_{(z, h(z))}=-z f^{\prime \prime}-h^{\prime}
$$

the role of assumption iv) on $f$ is to guarantee that consumption $c_{1}$ evaluated at the optimal points is an increasing function of $z$. Therefore we also have that the mapping $\theta(z)=c_{1}(z, h(z))$ has as inverse

$$
z=\theta^{-1}\left(c_{1}\right) \text {. }
$$

This allows us to translate the dynamics $h(z)$ into the consumption space This is stated in the following Lerma which is an elementary consequence of assumption $(v)$ on the production function $f$.

LEMMA. The points of the graph $\Gamma=((z, h(z)))$ of the function $x=$ $h(z)$ defined in the investment space are mapped by $\Psi$, which is defined by

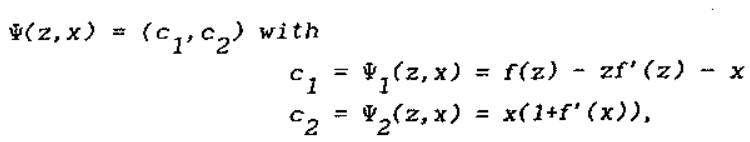

onto a curve $\Psi(\Gamma)$ contained in the consumption space. This curve is the graph of the function $c_{2}=\tau\left(c_{1}\right)$ expressing the relationship between consumptions at the points that we require to be optimal for the family of problems $P_{\boldsymbol{z}}$.

Note that $c_{2}=\tau\left(c_{1}\right)$ does not express the optimal consumption recurrence since it is not confugate of $x=h(z)$. The action of $\Psi$ can be seen in figure 2.

$\therefore$. Figure 2.

Before proceeding any further we explore the 1ssue of how wide is the fanily of production functions fulfilling assumptions 1), 1i) and i11) and iv) above.

THEOREM 2. Let $j$ be any integrable negative function $j: I \longrightarrow \mathbb{R}$ and let $J(\xi)$ be any third primitive of $j(\xi)$, that is, any function such that $J^{\prime \prime \prime}(\xi)=j(\xi)$. Then there exist real constants $c_{1}, c_{2}$ and $c_{3}$ such that $f(\xi)=J(\xi)+\lambda_{1} \xi^{2}+\lambda_{2} \xi+\lambda_{3}$ satisfies assumptions $i$ ), ii) iii) and $i v$ ) for every $\lambda_{1}, \lambda_{2}$; $\lambda_{3}$ such that $\lambda_{1}<c_{1} ; \lambda_{2}>C_{2} ; \lambda_{3}>C_{3}$.

This can be proved by checking that it works for the following values of $C_{1}, C_{2}, C_{3}$.

$c_{1}=\operatorname{mln}\left\{\inf \left\{-\frac{1}{2} J^{\prime \prime}(\xi): \xi \in I\right\}, \inf \left\{\frac{1}{2}\left(-\frac{h^{\prime}}{\xi}-J^{\prime}:(\xi)\right): \xi \in I\right\}\right.$

$c_{2}=\max \left\{\sup \left\{-J^{\prime}(\xi)-2 \lambda_{1} \xi ; \xi \in I\right\}\right.$,

$\left.\sup \left\{-1-\xi f^{\prime \prime}(\xi)-J^{\prime}(\xi)-2 \lambda_{1} \xi: \xi \in I\right\}\right\}$.

$C_{3}=\sup \left\{-J(\xi)-\lambda_{1} \xi^{2}-\lambda_{2} \xi: \xi \in I\right\}$. 
Observe that this characterises a wide family of production functions, parameterised by any arbitrary integrable negative function and the parameters $\lambda_{1}, 1 \leq 1 \leq 3$. For example, taking $J(\xi)=-6$ we get the family of polynomials

$$
\left\{-\xi^{3}+\lambda_{1} \xi^{2}+\lambda_{2} \xi+\lambda_{3}: \lambda_{1}<C_{1}, \lambda_{2}>C_{2} \text { and } \lambda_{3}>C_{3}\right\}
$$

With this we have specified fully the characteristics of the functions $h$ and $f$ that are taken parametrically in this approach. We now state the central 1ssue of this article. Let $x=h(z)$ be a function satisfying condition 1) above. Let $f$ be a production function satisfying assumptions i) to iv).

DEFINITION. We define $F_{h}$ to be the class of utllity functions such that for all $U \in F_{h}$ there exists a family of problems $\left(P_{z} J_{z \in I}\right.$ with utility function $U$ and production function $f$ such that the solution $x$ of each problem $P_{z}$ satisfies $x=h(z)$. We require all $U \in F_{h}$ to satisfy conditions a) to $e$ ) of section two.

PROBLEM: Characterise the class of functions $q_{h}$.

The basic idea behind the solution of this problem is very simple. We can impose specific slopes onto the indifference curves of a utility function $U$. which generates $h$ by defining a direction field (see Arnold (1974)). This field takes specific values at the points of the graph $\Psi(\Gamma)$ of $\tau\left(c_{1}\right)$ and has the indifference contours as integral curves. (See figure 3). We then analyse the relationship between the properties of these direction fields and those of the corresponding utillty functions. Finally we show that conditions over the direction fields that are easily manipulable allow us to construct an ordinal utility function, having the required propertles, that generates the given dynamics.

figure 3.

We start by defining the direction fleld associated to a function.

DEFINITION. Given $U: D \longrightarrow \mathbb{R}$, where $D$ is a domain in $\mathbb{R}^{2}$ and $U \in$ $C^{1}(D)$, we define the direction field $\Phi(U)$ associated to $U$ by

$$
\Phi(U)=Q\left(c_{1}, c_{2}\right)=-U_{1} N_{2} .
$$

Observe that $Q$ is well defined over the domain $D$ of $U$ if $U_{2}>0$ in $D$. This definition shows that $Q$ is a real function. He think of it as a direction field since it gives at each point $\left(\bar{c}_{1}, \bar{c}_{2}\right)$ of $D$ the slope of the contour curve of $U$ through that point. To see this let $U\left(c_{1}, c_{2}\right)=U\left(\bar{c}_{1}, \vec{c}_{2}\right)$ be the equation of such curve. If $U_{z}>0$ this equation implicitly defines $c_{2}$ in terms of $c_{1}$. We call this function $c_{2}=\varphi\left(c_{1}\right)$. We then have

$$
\varphi^{\prime}\left(c_{1}\right)=-U_{1} N_{2}=Q
$$

for all $\left(c_{1}, c_{2}\right)$ in a certain neighborhood of $\left(\bar{c}_{1}, \bar{c}_{2}\right)$. ( See figure 3$)$.

We now study the restriction of $\$$ to the family of utility functions $F$ Notice that for any two different functions $U$ and $V, \Phi(U)=\Phi(V)$ then $U$ and $V$ have common contour curves, since they are given by the first order differential equation (14). This does not imply that $U=V$ but it imposes an equivalence relation $U \simeq V \Leftrightarrow \Phi(V)=\Phi(U)$ induced by $\Phi$ over $F_{h}$ which identifies essentially equivalent utility functions.

DEFINITION. We define $V, W \in F_{\mathrm{h}}$ as essentially equivalent if there exists a differentiable, increasing, real function $\mu$ such that $V=\mu(W)$ in a 
certain open neighborhood of the set $\Psi(\Gamma)$ of optimal consumption points defined in the above lemma.

Therefore the equivalence classes induced in the set of utility functions by this equivalence relation are made up of utility functions giving an identical ordering to the 1ndifference contours. We now prove that the equivalence relation induced by $\Phi$ over $F$ is precisely the essential equivalence.

THEOREM 3. Let $V, W \in F_{h}$. Then $V$ and $W$ are essentially equivalent if and only if $\Phi(V)=\Phi(W)$ in a certain open neighborhood of $\Psi(\Gamma)$. ( The proof is given in the Appendix ).

We now show how the functions belonging to $F_{h}$ can be characterlsed by a certain property of their associated direction flelds.

THEOREM 4. Given a dynamics $\mathrm{x}=h(z)$ satisfying assumption 1) a utility function $U$ defined in an open neighborhood of $\Psi(\Gamma)$ belongs to $F$ if and only if its associated direction field $Q$ satisfies the first order quasi linear inequality in partial derivatives

$$
Q_{1}+Q_{2} Q \geq 0
$$

with an initial condition over $\Psi(\Gamma)$ given by

$$
Q\left(c_{1}, \tau\left(c_{1}\right)\right)=-g\left(c_{1}\right) \text {, }
$$

where $-g\left(c_{1}\right)$ is the slope of the budget constraints at the optimal points, which is given by $g\left(c_{1}\right)=A\left(h\left(\theta^{-1}\left(c_{1}\right)\right)\right)=A(x)$, given (7) and (9). (For a proof see Appendix.)

Theorem 4 states that every $Q$ verifying its hypotheses allows us to obtain a utility function $U$ that generates the dynamics $h$. The proof of this
Theorem suggests a way of constructing utility functions belonging to $F_{h}$. Before doing this we proceed to explore the problem of how wide is the family of direction fields $Q$ that verify the conditions of Theorem 4 above.

THEOREM 5. For every positive function $\eta$ defined over an open neighborhood of $\Psi(\Gamma)$ the quasi linear equation in partial derivatives

$$
Q_{1}+Q_{2} Q=\eta
$$

defines-a direction field $Q$ verifying (15) and (16) of theorem 4. (The proof is given in the Appendix).

We now describe a way to construct utility functions belonging to $F_{h}$ based on the previous two Theorems.

We know by (14) that a contour curve of a utility function $U$ with $U_{2}$ positive must satisfy the differential equation $\varphi^{\prime}\left(c_{1}\right)=Q\left(c_{1}, \varphi\left(c_{1}\right)\right)$. Taking derivatives with respect to $c_{1}$ we get $\varphi^{\prime \prime}\left(c_{1}\right)=Q_{1}+Q_{2} Q$. Taking a positive function $\eta$ as in Theorem 5 and $a Q$ that satisfies equation (17) the solutions of the second order differential equation $\varphi^{\prime \prime}=\eta$ with initial conditions $\varphi\left(c_{1}\right)=\tau\left(c_{1}\right)$ and $\varphi^{\prime}\left(c_{1}\right)=-g\left(c_{1}\right)$ give us the contour curves of $U$ in Theorem 5. The proof of Theorem 4 shows that if we define

$$
U\left(c_{1}, \varphi\left(c_{1}\right)\right)=c_{1}
$$

for alI the points in the contour curve 4 through $\left(c_{1}, \tau\left(c_{1}\right)\right)$, we obtain a utility function $U \in F_{\mathrm{h}}$. For example, if we take $\eta=1$ the contour curves of $U$ are clearly the family of quadratic functions $c_{1}^{2} / 2+\beta c_{1}+\alpha$. If $\eta=0$ we obtain the family of straight lines $\beta c_{1}+\alpha$. 


\section{CONCLUSIONS}

For a given $f$ verifying assumptions i) to iii) in section 2, the set $\mathcal{E}_{f}$ of economies analysed in the text is parameterised by the utility functions $u$ belonging to the class $u_{r}$ verifying assumptions a) to e). Theorem 1 proves the existence of a mapping $G: u_{f} \longrightarrow H$ assigning a unique $h$, belonging to the set $\mathcal{H}$ of dynamics verifying assumption 1 ) in section three, to each $U$ $u_{f}$

In theorem 2 we show that, for every h€H, there exists a wide family of conventional production functions $\mathcal{F}_{n}$ fulfilling assumptions i) to iv). Theorems 4 and 5 show that there exists a set $g=\left\{(h, f): h \in \mathcal{H}, f \in \mathcal{F}_{h}\right\}$ and a correspondence $J: € \longrightarrow u_{f}$ assigning to each $(h, f)$ in $g$ the utility functions generating, the dynamics $h$. This is a multivalued correspondence as a consequence of theorem 4. Moreover $G$ is such that $G(J(h, f))=h$ for all $(h, f) \in \mathscr{E}$. Some more information about the character of the correspondences $G$ and $J$ can be extracted from the text. He know that $G$ carries the class of economies corresponding to utility functions with non negative cross partial derivatives onto a set of increasing monotonic dynamics. To these dymamics there corresponds either a unique equillbrium or multiple equilibria without cycles. However the problem of finding the necessary conditions for an economy to generate a monotone dynamics remains open.

Theorems 4 and 5 describe the multivalued inverse correspondence $J$. More precisely, theorem 5 shows how the sets $J((h, f))$ characterised in theorem 4 can be parameterised by the class of non negative $c^{2}$ functions. Thus if we fix a function $\eta\left(c_{1}, c_{2}\right)$ belonging to such class we can define a mapping $J_{\eta}$ assigning to each $(h, f) \in \mathcal{S}$ the usique utility function in $J((h, f))$ that has $n\left(c_{1}, c_{2}\right)$ as the value of the second derivative of its contour curves. Hence, for fixed $f$ and $\eta$, there exists a bijective mapping between preferences and dynamics. Therefore in our model complete information on the dynamics does not identify individual preferences exactly. Due to the topological argument given in the introduction this may well be a generic tralt of all $O G$ models. However if we were to take the function $n$, as an extra assumption on $U$, thus taking the elasticity of substitution as a datum, an observed dynamics would give full information on preferences.

The flexibility of the model for generating a given dynamic leads to the following problem : could it be the case that if we take a class of dynamics as given ( for example the family $\mu h(x)$ for a fixed $h$, and $\mu$ taking values in some interval) there exist a unique $U$ and a family of production functions generating the entire class of dynamics?. Since the whole family of dynamics will impose restrictions on $U$ over an open domain of the plane 1t seems likely that the answer will be yes, when adequate restrictions are placed on the production functions.

In this article the production function plays always the role of a fixed parameter function. A natural problem of an inverse nature would be

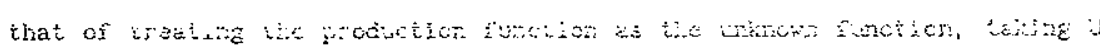
and $h$ as given. This seems to be a more awkward problem to analyse because it involves solving differential equations with deviating argument. 


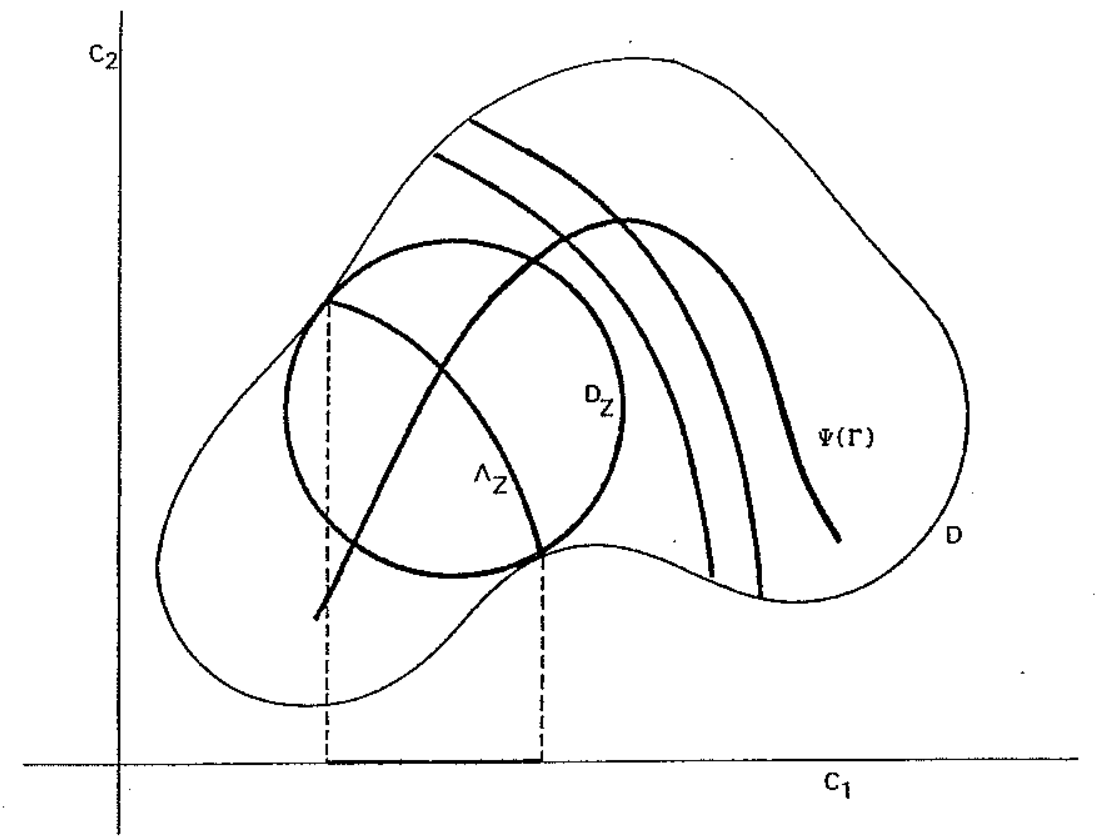

Figure 1. Some budget constralnts for the family $\mathbf{P}_{\mathbf{z}}$.

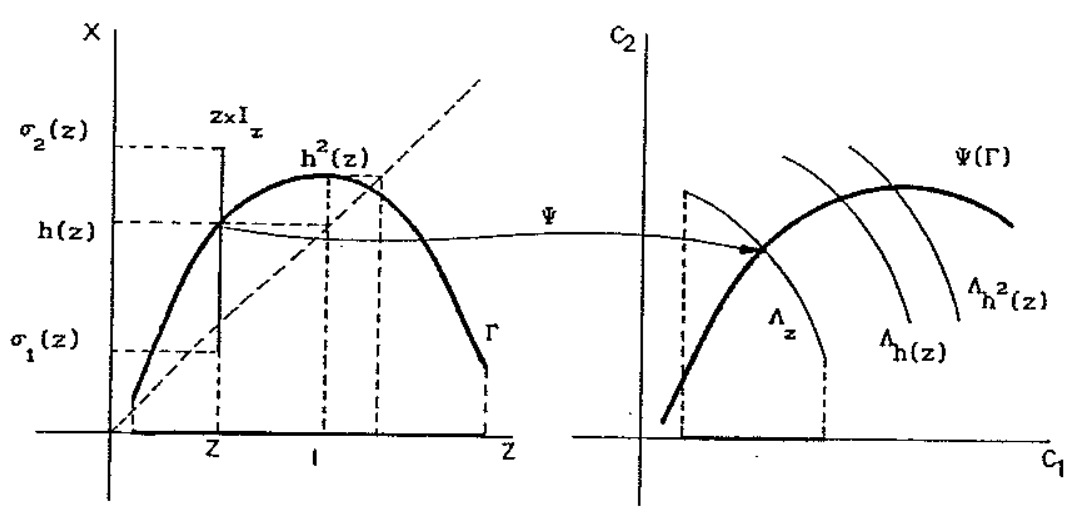

Figure 2. $\Psi$ waps the opt 1mal path $x=h(z)$ onto the consumption spaco. The budget constraint $z \times I_{z}$ of $P_{z}$ is mapped onto $\Lambda_{z}$. 


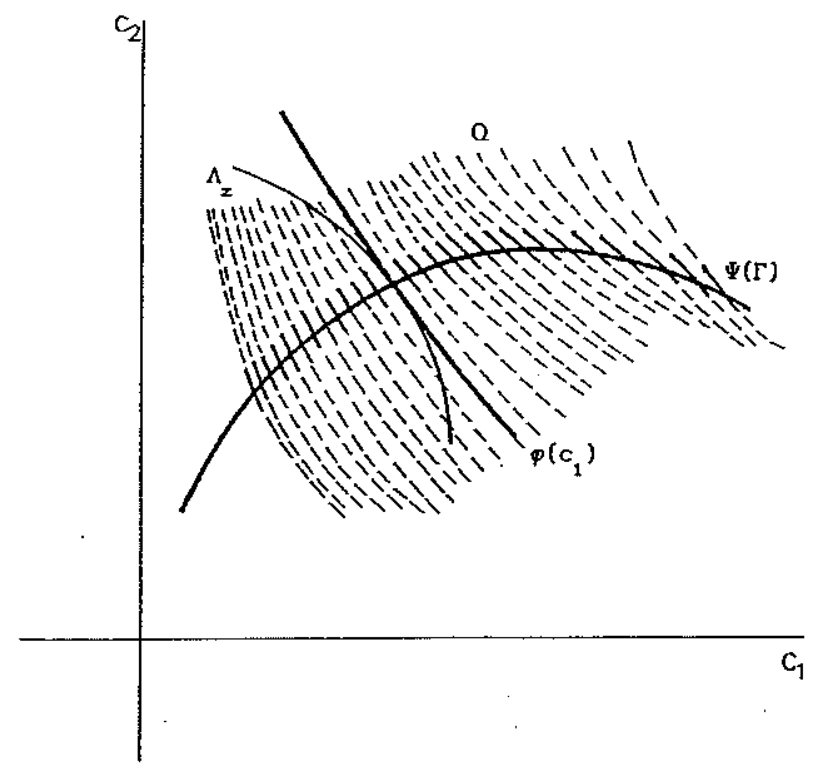

Flquic 3. The slopes lmposed onto $\Psi(\mathrm{N})$ can be extended to a direction fleld whose integral curves are the indifference curver of the solution $U$ to the inverise problem.

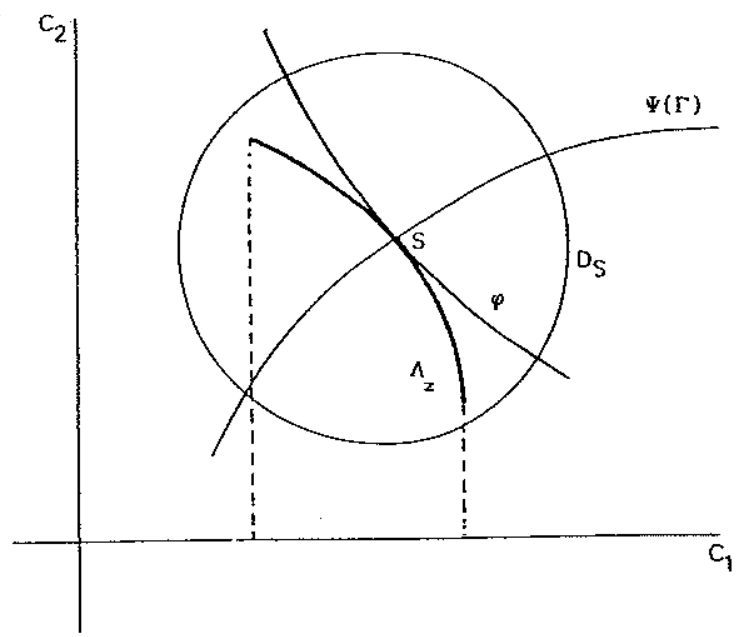

Figure 11. The bounds $\sigma_{1}$ and $\sigma_{2}$ restriet the set $\Lambda_{z}$ to the area in which $U$ is well deflned. 
Take now any polnt $P=\left(c_{1}^{*}, \dot{c}_{2}^{*}\right) \in D_{z}^{-}=D_{z} \cap \pi^{-}$and assume $U\left(c_{1}^{*}, c_{2}^{*}\right) \geq$ $U\left(c_{1}^{0}, c_{2}^{0}\right)$ : By virtue of the quasi concavity of $U_{4}$ for every $\left(c_{1}, c_{2}\right)$ belonging to the segment $\overline{P S}$ we would have $U\left(c_{1}, c_{2}\right) \geq U\left(c_{1}^{0}, c_{2}^{0}\right)$. But if we take $\left(c_{1}, c_{2}\right)$ Within a small enough distance of $S_{\text {, then }} c_{2}<\varphi\left(c_{1}\right)$, since by the quasi concavity of $U$ the function $\varphi$ is convex and in consequence $\varphi \geq L_{\mathrm{s}}$. Then $U\left(c_{1}, c_{2}\right)<U\left(c_{1}, \varphi\left(c_{1}\right)\right)$, since $U_{2}$ is strictly positive. But $U\left(c_{1}, \varphi\left(c_{1}\right)\right)=$ $U\left(c_{1}^{0}, c_{2}^{0}\right)$, and this contradicts our previous statement that $U\left(c_{1}, c_{2}\right) z$ $U\left(c_{1}^{0}, c_{2}^{0}\right)$. We therefore have proved the assumption $U\left(c_{1}^{*}, c_{2}^{*}\right) \geq U\left(c_{1}^{0}, c_{2}^{0}\right)$ to be absurd, and in consequence $U\left(c_{1}^{*}, c_{2}^{*}\right)<U\left(c_{1}^{0}, c_{2}^{0}\right)$ holds for every $\left(c_{1}^{*}, c_{2}^{*}\right) \in D_{z}^{-}$. To end our proof it suffices to observe that the concavity of $R$ implies that $n_{z}-\{S\} \subseteq D_{z}^{-}$

\section{Proof of the lemra.}

The restriction of $\Psi$ to $\Gamma$ is an injective mappling since two points of $\Gamma$ having the same inage must be identical. This can be easily proved. Assume $\Psi\left(z_{1}, h\left(z_{1}\right)\right)=\Psi\left(z_{2}, h\left(z_{2}\right)\right)$. Then

$$
\Psi_{1}\left(z_{1}, h\left(z_{1}\right)\right)=\Psi_{1}\left(z_{2}, h\left(z_{2}\right)\right)
$$

But given equation (10) this implies

$$
f\left(z_{1}\right)-z_{1} f^{\prime}\left(z_{1}\right)-h\left(z_{1}\right)=f\left(z_{2}\right)-z_{2} f^{\prime}\left(z_{2}\right)-h\left(z_{2}\right)
$$

which, given assumption iv) above, implies $z_{1}=z_{2}$. Therefore

$$
\left(z_{1}, h\left(z_{1}\right)\right)=\left(z_{2}, h\left(z_{2}\right)\right)
$$

Moreover the curve $\Psi(\Gamma)$ defines the graph of a function $c_{2}=\tau\left(c_{1}\right)$. To see this assume that two points in $\Psi(\Gamma)$ have equal first coordinate. Then $\Psi_{1}(z, h(z))=\Psi_{1}\left(z^{\prime}, h\left(z^{\prime}\right)\right)$ implies $z=z^{\prime}$. Therefore $(z, h(z))=\left(z^{\prime}, h\left(z^{\prime}\right)\right)$ and $\Psi_{2}(z, h(z)\}=\Psi_{2}\left(z^{\prime}, h\left(z^{\prime}\right)\right)$. This shows that both points colncide. q.e.d. 
Proof of theorem 2.

Proof. Since $f^{\prime \prime}=J^{\prime \prime}=j<0$ assumption ili) is verifled by $f$. We take $C_{1}$ such that

$c_{1}=\min \left\{\operatorname{lnf}\left\{-\frac{1}{2} J^{\prime \prime}(\xi): \xi \in I\right), \operatorname{lnf}\left\{\frac{1}{2}\left(-\frac{h^{\prime}}{\xi}-J^{\prime \prime}(\xi)\right): \xi \in I\right\}\right.$ Since $f^{\prime \prime}(\xi)=J^{\prime \prime}(\xi)+2 \lambda_{1}$, if $\lambda_{1}<c_{1}$ then $f^{\prime \prime}<0$ and $f^{\prime \prime}<-\frac{h^{\prime}}{\xi}$ for every $\xi \in I$, which is equivalent to $1 v$ ).

Let

$C_{2}=\max \left\{\sup \left\{-J^{\prime}(\xi)-2 \lambda_{1} \xi: \xi \in I\right\}\right.$,

$\left.\sup \left\{-1-\xi f^{\prime \prime}(\xi)-J^{\prime}(\xi)-2 \lambda_{1} \xi: \xi \in I\right\}\right\}$.

Since $f^{\prime}(\xi)=J^{\prime}(\xi)+2 \lambda_{1} \xi+\lambda_{2}$, if $\lambda_{2}>C_{2}$ then $f^{\prime}>0$ and $1+f^{\prime}+\xi f^{\prime \prime}>0$. Finally take $C_{3}$ such that

Then, if $\lambda_{3}>C_{3}, f>0$.

$$
c_{3}=\sup \left\{-J(\xi)-\lambda_{1} \xi^{2}-\lambda_{2} \xi: \xi \in I\right\}
$$

Note that the family verifying only assumptions i), ii) and 1i1), excluding iv), is even wider than the class we have found. It can be obtained by relaxing the constant $C_{1}$ to

$$
\mathrm{C}_{1}=\inf \left\{-\frac{1}{2} \mathrm{~J}^{\prime \prime}(\xi): \xi \in \mathrm{I}_{\mathrm{z}}\right\}
$$

PROOF, OF THEOREM 3. If $V$ and $W$ are essentially equivalent then $v_{1}=$ $\mu^{\prime} W_{1}$ and $V_{2}=\mu^{\prime} W_{2}$ in an open nelghborhood of $\Psi(\Gamma)$. Since $\mu^{\prime}>0, \Phi(V)=$ $\Phi(W)$ in that nelghborhood.

Assune now that $\Phi(V)=\Phi(W)=Q$ in an open set $D^{\prime} \supset \Psi(r)$. Then $V$ and $W$ are solutions of the first order linear equation in partial derivatives

$$
U_{1}+U_{2} Q=0
$$

By virtue of the Theorem of existence and uniqueness of solutions for these equations ( see Arnold (1980)), given $s=\left(c_{1}, \tau\left(c_{1}\right)\right) \in \Psi(\Gamma)$ there exists a certain neighborhood $D_{s}$ of $S$ in which $V$ and $W$ are uniquely defined by their values over $\Psi(\mathrm{r}) \cap D_{s}$, provided the characteristic curves of the equation (A1), which are the common contour curves of $U$ and $W$, are transversal (non tangent $)$ to $\Psi(\Gamma)$. That $1 \mathrm{~s}$, provided

$$
\tau^{*}\left(c_{1}\right) \neq \varphi^{*}\left(c_{1}\right)
$$

where $\varphi\left(c_{1}\right)=c_{2}$ is the contour curve of $U$ and $W$ through $\left(c_{1}, \tau\left(c_{1}\right)\right)$. Since $V, W \in F_{h}$, every point $s=\left(c_{1}, \tau\left(c_{1}\right)\right) \in \Psi(\Gamma)$ satisfies the first order condition of the problem $P_{z}$, where $z=\theta^{-1}\left(c_{1}\right)$. Therefore

$$
-U_{1}+U_{2} g\left(c_{1}\right)=0,
$$

where $\mathrm{g}\left(\mathrm{C}_{1}\right)=\mathrm{A}\left(\mathrm{h}\left(\theta^{-1}\left(\mathrm{C}_{1}\right)\right)\right.$ ). Hence (13) and (A3) imply

But from (14) we get

$$
Q\left(c_{1}, \tau\left(c_{1}\right)\right)=-g\left(c_{1}\right)
$$

$$
\varphi^{\prime}\left(c_{1}\right)=-g\left(c_{1}\right)
$$

Thus a sufficient condition for transversality is

$$
\tau^{\prime}\left(c_{1}\right)+g\left(c_{1}\right)>0
$$

To prove that this inequality holds, observe that $\tau^{\prime}\left(c_{1}\right)$ gives the slope of 
the image of the given recurrence in the consumption space. The points $(z, h(z))$ must satisfy the budget constralnts (2) and (3) after substituting $h(z)$ by $x$. In that case

$$
\tau^{\prime}\left(c_{1}\right)=\frac{h^{\prime}(z)\left(1+f^{\prime}(x)+x f^{\prime}(x)\right)}{-z f^{\prime \prime}(z)-h^{\prime}(z)}=\frac{h^{\prime}(z) g\left(c_{1}\right)}{-z f^{\prime}(z)-h^{\prime}(z)} .
$$

Therefore

$$
\tau^{\prime}\left(c_{1}\right)+g\left(c_{1}\right)=\frac{-g\left(c_{1}\right) z^{\prime \prime}(z)}{-z f^{\prime \prime}(z)-h^{\prime}(z)} .
$$

which is positive given assumptions 1 ), i1) and $(v)$ above. Therefore $V$ and $W$ are uniquely defined over a certain nelghborhood $D_{s}$ of $s$ by (A1) and their Initial conditions

$$
\begin{aligned}
& \alpha\left(c_{1}\right)=V\left(c_{1}, \tau\left(c_{1}\right)\right) \\
& \beta\left(c_{1}\right)=W\left(c_{1}, \tau\left(c_{1}\right)\right)
\end{aligned}
$$

respectively. Taking derivatives we get

$$
v_{1}+v_{2} \tau^{\prime}=\alpha^{\prime}
$$

Since $V$ is a solution of (A1) and (A4) holds we have

$$
v_{1}-v_{2} g\left(c_{1}\right)=0 \text {. }
$$

Therefore

$$
\mathrm{V}_{2}\left(g+\tau^{\prime}\right)=\alpha^{\prime}
$$

But $V_{2}>0\left(\right.$ since $\left.V \in F_{b}\right)$ and $g+\tau^{\prime}>0$ by (A5). Therefore $\alpha^{\prime}>0$. A sintlar reasmbe phows tha: $\beta^{2}>0$. If ite take $\mu=\alpha \circ \beta^{-1}$ then $\mu^{\prime}>0$ and $\mu$ oh is a solution of (A1) which takes the same values over $D_{s} \cap \Psi(\Gamma)$ as $v$. This proves that $v=\mu$ of $W$ over $D_{s}$ and also over $D=U_{s \in \Psi}(\Gamma) D_{s}$ q.e.d.
PROOF OF THEOREM 4. a) He assume first that $U \in \mathcal{F}_{h}$ and prove that $Q$ satisfies the required conditions. If in the expression $Q_{1}+Q_{2} Q$ we make $Q=$ $-U_{1} \sim_{2}$ we obtaln

$$
Q_{1}+Q_{2} Q=\frac{-U_{11} U_{2}^{2}+2 U_{21} U_{1} U_{2}-U_{22} U_{1}^{2}}{\left(U_{2}\right)^{3}} .
$$

But the quasi concavity of $U$ implles that

$$
0 \leq-u_{11} u_{2}^{2}+2 u_{21} u_{1} u_{2}-U_{22} u_{1}^{2}=\operatorname{det}\left[\begin{array}{ccc}
u_{11} & u_{12} & u_{1}^{1} \\
u_{21}^{2} & u_{22}^{12} & v_{2}^{2} \\
u_{1} & u_{2}^{2} & 0^{2}
\end{array}\right] \text {. }
$$

Therefore $U_{2}>0$ implles

$$
Q_{1}+Q_{2} Q \geq 0
$$

Also since $h$ picks interior solutions to $P_{z^{\prime}}$ equation (A4) guarantees that the initial condition holds. q.e.d.

b) We now assume that $Q$ verifies conditions (15) and (16) in an open neighborhood of $\Psi(\Gamma)$. We prove first that there exists a function $U$ whose associated field is $Q$ and such that $U_{1}, U_{2}>0$. To do this notice that the Theorem of existence and uniqueness of solutions of first order linear equations in partial derivatives guarantees, for every $s \in \Psi(\Gamma)$, the existence, in an open ball $D$, centered at $s$, of a unique solution for the Cauchy problem

$$
U_{1}+U_{2} Q=0
$$

with infial condition over $\Psi(\Gamma)$ given by

$$
U\left(c_{1}, \tau\left(c_{1}\right)\right)=c_{1}
$$

provided that the transversallty condition holds. To prove that we take dertvatives in equation (A8). We then get

$$
U_{1}+U_{2} \tau^{\prime}=1
$$

which given (16) and (A7) implies 


$$
\mathrm{U}_{2}\left(\tau^{\prime}+g\right)=1
$$

Therefore $U_{2}>0$ at $s$ since, given (A5) above, $\tau^{\prime}+g>0$. But $Q<0$ at $s$. Hence (A7) implies that $U_{1}>0$ at $s$ and, by the continuity of $U_{1}$ and $U_{2}$, these functions are strictly positive in a certain open ball $D_{\mathrm{g}}$ centered at s. The uniqueness of the solution at every $D_{B}$ guarantees that any two solutions obtained for two different points $s$ and $s^{\prime}$ must be the same at $D_{\mathrm{s}} \mathrm{D}_{\mathrm{s}}$. Hence $U$ is well defined in the open nelghborhood $D$ of $\Psi(r)$ given by $U_{s \in \Psi(\Gamma)} \dot{D}_{s}$. Moreover, since $Q$ is $C^{2}$, the Theorem mentioned above guarantees that $U$ is also $C^{2}(D)$.

We have proved that $U$ satisfies conditions a), b) and c) of Section two. We now prove that $U$ is quasi concave in each $D_{g}$. Since $U_{2}>0$ at every $\left(\bar{c}_{1}, \bar{c}_{2}\right) \in D_{s}$, the equation $U\left(c_{1}, c_{2}\right)=U\left(\bar{c}_{1}, \bar{c}_{2}\right)$ implicitly defines $c_{2}$ as a function $\varphi\left(c_{1}\right)$. Taking derivatives with respect to $c_{2}$ in (14) we find $\varphi^{\prime \prime}=$ $Q_{1}+Q_{2} Q \geq 0$. Hence the indifference curves of $U$ are convex. Since $U_{1}$ and $U_{2}$ are positive this guarantees that $U$ is quasi concave in $D_{s}$.

Finally we prove that $U$ generates the given dynamics for a certain family of problems with production function $f$. To do this we $f 1 x$ a value $z$ belonging to the domain $I$ of $h$. The point $\Psi(z, h(z))=s$ belongs to the open ball $D_{s}$ in which $U$ is well defined. We consider two values $\sigma_{1}(z)$ and $\sigma_{2}(z)$ such that

$$
\sigma_{1}(z)<h(z)<\sigma_{2}(z)
$$

and the budget set $\Lambda_{z}$ defined in (5) verifies

$$
\Lambda_{z} \leq D_{s}
$$

(See figure A1).
Also every $x=h(z)$ verifies the first order condition ( this is implied by the initial condition for $Q$ in (16) together with (A7)). Hence the hypotheses of Theorem 1 are satisfied by $U$ and $f$. Therefore $U$, together with $f$ generates, the given dynamics q.e.d.

\section{Figure A1.}

PROOF OF THEOREM 5 Consider the quasi linear equation in partial derivatives $Q_{1}+Q_{2} Q=\eta$ with initial condition over $\Psi(\Gamma)$ given by (16).

The transversality condition requires the initial curve, whose equation parameterised by $c_{1}$ are

$$
\therefore \quad x_{1}=c_{1} ; x_{2}=\tau\left(c_{1}\right) ; x_{3}=-g\left(c_{1}\right) \text {. }
$$

to be transversal to the integral curves of the associated differential system

$$
d x_{1}=\frac{d x_{2}}{x_{3}}=\frac{d x_{3}}{\eta}
$$

The direction vector of the initial curve is $\left(1, \tau^{\prime},-g^{\prime}\right)$ and the direction vector of a characteristic curve through a point of the initial curve is $\left(1,-g\left(c_{1}\right), \eta\left(c_{1}, \tau\left(c_{1}\right)\right)\right.$. Therefore (A5) guarantees the transversality condition for this equation at every point of the initial curve.

By virtue of the Theorem of existence and uniqueness of solution for these kind of equations a solution $Q$ of (17) verifying (16) exists in an open neighborhood of $\Psi(\Gamma)$, and since $\eta>0$ we have

$$
Q_{1}+Q_{2} Q>0
$$

q.e.d. 
References.

Arnold, V. A. (1974), Equations Différentielles Ordinaires, (Moscou MIR).

Arnold, V. A. (1980), Chapitres Supplémentaires de la Théorie des Equations Différetielles, (Moscou MIR).

Benhabib, J. and R. Day (1982), "A Characterization of Erratic Dynamics in the Overlapping Generations Model," Journal of Economic Dynamics and Control, 4, 37-55.

Benhabib, J. and K. Nishimura (1985) , "Competitive Equilibrium Cycles," Journal of Economic Theory, 35, 284-306.

Boldrin, $M$. and L. Montrucchio (1986), "On the Indeterminacy of Capital Accumulation Paths," Journal of Economic Theory, 40, 26-39.

Debreu, G. (1974), "Excess Demand Functions" Journal of Mathematical Economics, 1, 15-23.

Deneckere, R and S. Pelikan (1986), "Competitive Chaos," Journal of Economic Theory, 40, 13-25.

Falconer K. (1990), Fractal Geometry. (John Wiley).

Grandmont, J. M. (1985), "On Endogenous Competitive Business Cycles," Econometrica, 5, 995-1044.

Li, T. and J. Yorke (1975), "Period Three Implies Chaos," American Mathematical Monthly, 82, 985-992.

Madden, P. (1986), Concavity and Optimization in Microeconomics, (Oxford University, K., Blackwell).

Mantel, R. R. (1974), "On the Characterization of Aggregate Excess Demand", Journal of Economic Theory, 7, 348-353.

Sorger, G. (1992) "On the Minimum Rate of Impatience for Compllcated Optimal Growth Paths", Journal of Economic Theory, 56, 160-179. 